\title{
RELACIÓN ENDOPERIODONTAL VERDADERA 0 CLASE III Y TRATAMIENTO REGENERATIVO. REPORTE DE UN CASO
}

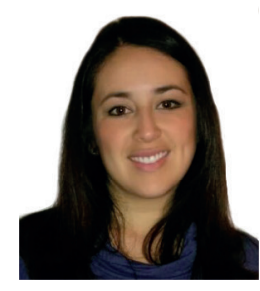

ODONT. KATHERRNE A. ROMERO ESPNNOZA ESPECIALISTA EN PERIODONCIA PROFESORA DE LA UNIVERSIDAD DE CUENCA

\section{RESUMEN}

Dentro de la práctica odontológica existen pocas especialidades tan íntimamente relacionadas como son la Endodoncia y la Periodoncia, ya que tanto el tejido pulpar como el periodonto comparten una relación embriológica, anatómica, microbiológica y patológica.

Las lesiones endoperiodontales, son una condición en las que la pulpa y el periodonto están interrelacionadas, manifestándose en ello pérdida de inserción periodontal, destrucción de hueso y periodontitis periapical.

Al realizar la terapia periodontal el objetivo es la resolución de la afección en los tejidos de soporte del diente. La terapia endodóntica trata la enfermedad, los tejidos periapicales y pulpares afectados.

El objetivo de este trabajo es presentar un caso clínico de lesión endoperiodontal verdadera, en el que se realizó inicialmente un tratamiento endodóntico, seguido del tratamiento periodontal con regenerativo de un defecto infraóseo utilizando biomateriales, específicamente membrana reabsorbible de colágeno y hueso bovino liofilizado.

El seguimiento a un año indica resolución del defecto óseo, estabilidad dental, ausencia de enfermedad periodontal, lo que indica éxito en el tratamiento. 


\section{INTRODUCCIÓN}

Las lesiones endoperiodontales son aquellas que incluyen la interacción de la enfermedad pulpar y periodontal. Desde el desarrollo del germen dental se forman estructuras como son los túbulos dentinarios y los conductos principales, secundarios, laterales e interradiculares, capaces de comunicar estructuras endodónticas con periodontales razón por la cual es común que una enfermedad pulpar afecte las estructuras periodontales y viceversa. Las relaciones entre la enfermedad pulpar y periodontal ocurren a través de las íntimas conexiones anatómicas y vasculares que existen entre la pulpa y el periodonto.(1)

En el desarrollo embriológico el tejido mesenquimático se divide en elementos epiteliales del germen que confluyen en el área apical: saco dental y papila. Esta confluencia hace que compartan tejidos vasculares, neurales y fibras conectivas tanto para el ligamento periodontal cuanto para el tejido pulpa rapical; (2)esto equivale a tener similar inervación, irrigación y drenaje linfático; razón por la cual no llama la atención la relación patológica entre las dos entidades.(3)

Al momento del desarrollo radicular se forman otras puertas de comunicación: foramen apical, conductos laterales, accesorios, y túbulos dentinarios, siendo vías a través de las cuales los factores etiológicos pueden afectar la pulpa y el periodonto.(4)

Sin embargo, muchas veces tanto la enfermedad como el tratamiento de las lesiones endodónticas y periodontales pueden progresar independientemente una de la otra, pero en otras ocasiones la estrecha relación endodóntica periodontal debe ser reconocida y tratada en ambos aspectos como una enfermedad combinada y así lograr una resolución exitosa de la lesión. (5)

Según el origen de la patología las lesiones endoperiodontales se pueden clasificar en:

Lesión Endodóntica Primaria Periodontal Secundaria:en este caso se inicia en un proceso periapical de origen pulpar que puede drenar hacia el surco gingival simulando ser una lesión de origen periodontal.(6) La pulpa necrótica puede producir un tracto fistuloso desde el ápice a través del periodonto hacia el borde gingival destruyendo el hueso alveolar.(7)El paciente puede manifestar mínimo malestar; radiográficamente los diferentes niveles de pérdida ósea dependen del tracto de la fístula. La fístula transperiodóntica se manifiesta clínicamente como una bolsa periodontal profunda y angosta que llega hasta el ápice.(8)

El tratamiento de elección es la terapia endodóntica.Está indicado realizarlo antes de la terapia periodontal. El tratamiento puede esclarecer el diagnóstico puesto que si sede la bolsa al concluir el tratamiento endodóntico la lesión tenía origen pulpar.(9)

Lesión Periodontal Primaria, Endodóntica Secundaria: A medida que las lesiones periodontales progresan hacia el ápice, los conductos laterales o accesorios pueden estar expuestos a un ambiente injurioso, pudiendo conducir a necrosis pulpar. $(8,10)$

Las necrosis pulpares pueden degenerarse a partir de la terapia periodontal, con la cual es posible seccionar los paquetes vasculonerviosos que entran a través de los conductos secundarios, accesorios y el foramen apical, los cuales nutren la pulpa. $(8,11)$

Estas lesiones radiográficamente pueden ser no visibles.

Siempre que no responda a la terapia periodontal se debe evaluar la vitalidad, puesto que en el transcurso del tratamiento la fálta de vitalidad puede manifestarse, siendo necesario el tratamiento endodóntico.(11)

Las lesiones combinadas o lesiones Endoperiodontales Verdaderas: Se han descrito las lesiones endoperiodontales o pulpoperiodontales como aquellas lesiones de carácter inflamatorio que comprometen simultáneamente a la pulpa dental y a las estructuras del periodonto de inserción.(9) 
Esta combinación de características es considerada como una verdadera lesión endoperiodontal y el tratamiento incluye tanto la terapia pulpar como periapical; el grado de pérdida de la adherencia epitelial es invariablemente grande y el pronóstico reservado. $(12,13)$

Concluyendo, la lesión combinada se produce cuando ambas enfermedades coexisten en una misma pieza dentaria y se comunican entre sí.

Uno de los tratamiento requeridos en el caso de lesiones endoperiodontales es la regeneración ósea guiada (ROG), puesto que la afección de las dos entidades patológicas da como resulta- do pérdida ósea de soporte que muchas veces puede ser extensa. En virtud de esto se plantea la posibilidad de intervenciones quirúrgicas que permitan devolver el soporte óseo en los casos que amerite.

Este trabajo tiene como objetivo presentar un caso clínico de lesión endoperiodontal combinada, con tratamiento regenerativo, enfatizando en aquellos aspectos importantes para el diagnóstico y tratamiento.

zona periapical. Todos los datos clínicos y radiográficos indican compatibilidad con una lesión endoperiodontal combinada.(Figura 1)

a consulta odontológica a clínica privada, y es derivado al periodoncista por presentar movilidad y supuración a través del surco gingival en la zona mesial de la pieza 1.3. El paciente no refiere antecedentes médicos importantes, pero menciona que por motivos de trabajo no mantiene un horario establecido para cumplir con sus hábitos de sueño, alimentación e higiene. No ha recibido tratamiento periodontal previo.

El examen clínico demuestra la presencia de Periodontitis Severa Generalizada, ya que se registran profundidades de sondaje en promedio de más de $6 \mathrm{~mm}$, y pérdida de inserción de $5 \mathrm{~mm}$ en adelante. Concretamente la pieza 1.3 presenta profundidad de sondaje por mesio vestibular de $11 \mathrm{~mm}$, por mesio palatino de $9 \mathrm{~mm},(-$ figura 1) adicionalmente sangrado al sondaje y supuración. La movilidad se registra como tipo II (Miller 1950) y adicionalmente no responde a las pruebas de sensibilidad al frío, hay que mencionar que la pieza además es pilar de una prótesis fija (PF) con extensión de 4 pónticos (extensión de 1.3 a 2.3). La P.F se encontraba desadaptada y en malas condiciones, lo cual podría involucrar la vitalidad pulpar.

La PF fue desmontada facilitando el examen clínico, posterior a esto el tramo fue provisionalizado. Al examen radiográfico se observó una lesión infraósea envolvente, comprometiendo la
El objetivo del tratamiento fue en primer lugar, el tratamiento endodóntico de la pieza 1.3, el mismo que fue realizado por la especialista en endodoncia, seguido a esto el paciente recibió tratamiento periodontal basado en raspado y alisado radicular generalizado, controlando conjuntamente todos aquellos factores de riesgo para la periodontitis, motivación y adiestramiento intra y extraoral.

Tras una primera fase de terapia básica periodontal, se esperó el tiempo de cicatrización de la bolsa, constatando la ausencia de supuración. La evaluación periodontal se ejecutó a los 21 días pos-terapia inicial, y los valores de sondaje presentados en ese momento fueron de $7 \mathrm{~mm}$ mesio vestibular y $5 \mathrm{~mm}$ mesio palatino.

Una semana después se procedió con la cirugía de regeneración ósea guiada; en este caso se realizó una incisión intrasurcular de mesial de la 1.3 hasta distal de la pieza 1.4. Se elevó un colgajo mucoperióstico a espesor total que dejó al descubierto un defecto infraóseo de dos paredes extendiéndose por debajo del ápice dental y envolviendo la zona.

Se realizó raspado y alisado radicular y acondi- 
cionamiento radicular de la superficie radicular con tetraciclina.

Tras el desbridamiento del defecto óseo, se regeneró mediante un xenoinjerto de hueso bovino liofilizado y una membrana de colágeno reabsorbible.

Se suturó con nylon 5/0 llevando a cabo puntos simples y un colchonero horizontal con el fin de evitar tensión en el colgajo. Finalmente se realizó una ferulización con el objetivo de disminuir la movilidad de la pieza durante la cicatrización.

Los cuidados postoperatorios incluyeron el cese de todo procedimiento para el control mecánico de la placa dental durante quince días, tiempo durante el cual el paciente realizó enjuagues con clorhexidina al $0,12 \%$ tres veces al día; dieta sana, evitar ejercicio físico, compresas frías las primeras 24 horas, además medicación analgésica y antibiótica.

\section{DISCUSIÓN Y CONCLUSIONES}

La lesión endodóntica-periodontal es una manifestación clínica de la intercomunicación patológica / inflamatoria entre el tejido pulpar y periodontal a través de estructuras tales como foramen apical, conductos laterales, conductos accesorios y túbulos dentinarios (14). Sobre la base de la patológica, Simon et al (8). clasificó las lesiones en: lesiones endodónticas primarias, lesiones endodónticas primarias con lesiones periodontales secundarias; lesiones periodontales primarias, lesiones periodontales con endodonticas secundarias, y lesiones verdaderas o lesiones combinadas.

Las lesiones endoperiodontales combinadas se manifiestan como una enfermedad periodontal que subyace con una enfermedad endodóntica o una patología endodóntica con una enfermedad periodontal, esto indica la íntima relación entre la pulpa dental y el periodonto.(15)Las lesiones combinadas requieren tanto tratamiento de endodoncia como tratamiento periodontal
Se realizó control a los 7, y a los 15 días se retiró la sutura. Se citó al paciente para un nuevo control al mes, momento en el que se observó una epitelización completa de los tejidos.

A los seis meses se visualizó radiográficamente una cicatrización completa del defecto (Figura 2). Clínicamente se observó una reducción de la profundidad de sondaje a $3 \mathrm{~mm}$ por vestibular y palatino y una ganancia de inserción. Finalmente, al año se constató la estabilidad de los resultados radiográficos y clínicos.

El paciente posteriormente fue tratado por el especialista en Rehabilitación Oral, quien rehabilitó la zona de las piezas 1.3 a la 2.3. Los resultados obtenidos han mejorado el pronóstico del diente, pero su estabilidad a largo plazo dependerá fundamentalmente del control de placa y del cumplimiento por parte del paciente del mantenimiento, el mismo que fue recomendado realizarlo trimestralmente. En la actualidad se mantienen controles periódicos periodontales, y protésicos.

y regenerativo, como se informa en este caso (14).

El tratamiento de las lesiones endoperiodontales combinadas son un verdadero desafío, ya que ellas exigen conocimientos profundos sobre curación tanto endodóntica como periodontal. (16)

Kim et al, (11) informaron que incluso con la ayuda de un microscopio, el tratamiento exitoso de lesiones endodóntica-periodontal combinada es significativamente menor que el tratamiento de las lesiones endodónticas aisladas.De 40 lesiones endodónticas-periodontales combinadas tratadas en su estudio, sólo el 57\% mostró una curación completa.(17)

Sin embargo la regeneración de un nuevo aparato inserción es uno de los aspectos más desafiantes de la terapia. La regeneración periodontal se basa en el tratamiento de defectos periodontales, y es lo vanguardista hoy en día en la investigación periodontal. El objetivo es re- 
generar los componentes periodontales que se han perdido a través de las lesiones endoperiodontales.

El uso de sustitutos óseos, y membranas, permiten una regeneración ósea guiada (15).

Se han propuesto varias modalidades de tratamientos para tratar dientes periodontalmente involucrados.

Los procedimientos regenerativos se indican en defectos intraóseos. Los procedimientos utilizados en el presente caso incluyen:xenoinjerto óseo bovino con características osteoinductivas y osteoconductivas, que rellena físicamente los defectos óseos, proporcionando un andamio para la formación ósea, el material óseo posee componentes orgánicos evitando reacciones alérgicas además está clínicamente bien tolera- do; al llenar el defecto, también impide el colapso de los tejidos blandos en el defecto óseo y la porosidad facilita la estabilización del coágulo sanguíneo facilitando el crecimiento de nuevos vasos (18). Se usó en combinación con membrana de colágeno reabsorbible.

Los resultados del presente caso sugieren que el injerto óseo y membrana de colágeno ayudan a la regeneración de defectos infraóseos.

Sin embargo es indispensable tener presente que hace falta realizar un diagnóstico minucioso de las entidades tanto periodontales como endodónticas y establecer un adecuado plan de tratamiento que se oriente a restituir la salud periodontal y la estabilidad endodóntica, sin dejar de lado la posibilidad de regenerar los tejidos de soporte; práctica que hoy en día está clínicamente comprobada y nos ofrece un pronóstico adecuado para las piezas dentales tratadas.

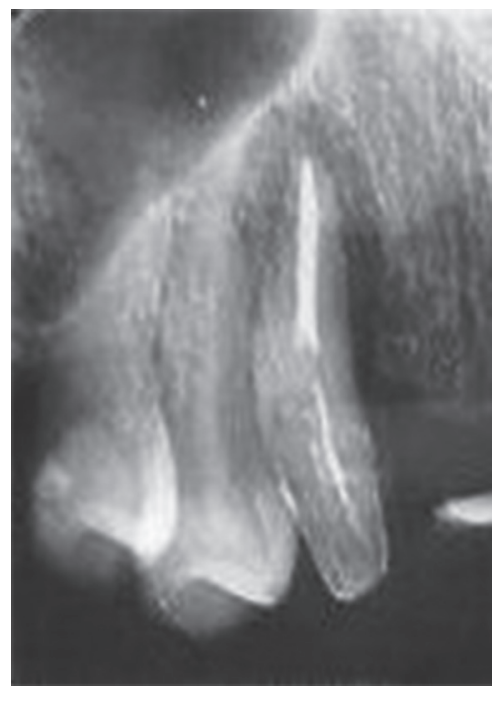

FIG 1:

Se observa en la imagen pieza

1.3 con tratamiento de conducto, la misma que presenta perdida osea envolvente por mesial.

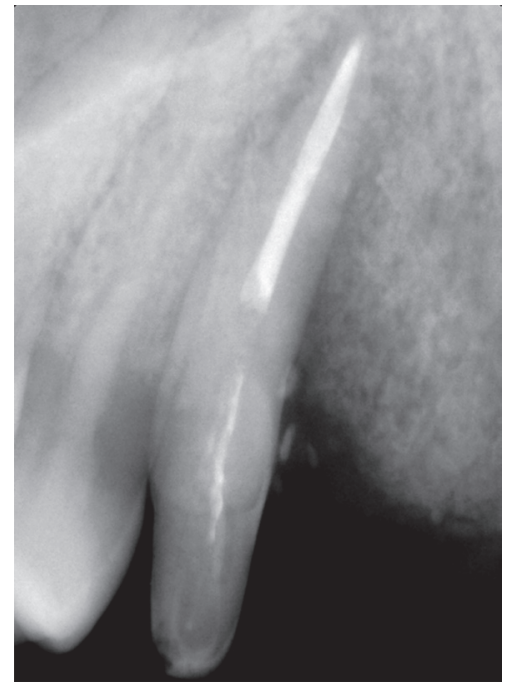

FIG 2:

Pieza 1.3 sometida a Regeneracion Ósea Guiada en la que se uso xenoinjeto y membrana de colageno. 


\section{BIBLIOGRAFÍA}

1. Hargreaves KM, Berman LH, Cohen S. Cohen. Vías de la Pulpa + ExpertConsult: Elsevier Health Sciences Spain; 2011.

2. Stallard RE. Periodontic-endodontic relationships. Oral Surgery, Oral Medicine, Oral Pathology. 1972;34(2):314-26.

3. Basrani E, Cañete MT, Blank AJ. Endodoncia integrada: Actualicdades Médico Odontológicas Latinoamérica; 1999.

4. Teniente 0 , Yáñez I. Relación endoperiodontal: diag-nóstico, tratamiento y pronóstico. Rev Mex Odon Clín. 2008;2(2):22-4.

5. Simon P, Jacobs D. The so-called combined periodontal-pulpa problem. Dental Clinics of North America. 1969;13(1):45-52

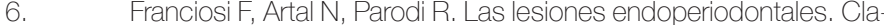
ves odontol. 1997;4(26):3-6, 8-12.

7. Simring M, Goldberg M. The pulpal pocket approach: retrograde periodontitis. Journal of Periodontology. 1964;35(1):22-48.

8. Simon JH, Glick DH, Frank AL. The relationship of endodontic-periodontic lesions. Journal of periodontology. 1972:43(4):202-8.

9. $\quad$ Acevedo MV, Machado MDL. Sociedad de Endodoncia Latino Americana SELA. Revista de la Sociedad de Endodoncia de Chile.50.

10. Rubach WC, Mitchell DF. Periodontal disease, accessory canals and pulp pathosis. Journal of Periodontology. 1965;36(1):34-8.

11. Blair HA. Relationships between endodontics and periodontics Journal of periodontology. 1972:43(4):209-13.

12. Huynh-Ba G, Kuonen P, Hofer D, Schmid J, Lang NP, Salvi GE. The effect of periodontal therapy on the survival rate and incidence of complications of multirooted teeth with furcation involvement after an observation period of at least 5 years: a systematic review. Journal of clinical periodontology. 2009;36(2):164-76.

13. Moreno García N, Todd Jiménez M, Téllez Jiménez H, Salazar Lozano S, Guerrero Del Ángel F. Lesiones endoperiodontales de gran tamaño. Uso del plasma rico en factores de crecimiento combinado con aloinjerto y membranas de fibrina autóloga. Revista Nacional de Odontología [serie en internet]. 2010;2

14. Verma PK, Srivastava R, Gupta K, Srivastava A. Combined endodontic-periodontal lesion: A clinical dilemma. Journal of Interdisciplinary Dentistry. 2011;1(2):119.

15. Ari G, Kumar KA, Ramakrishnan T. Treatment of an intrabony defect combined with an endodontic lesion: a case report. Endodontic Practice Today. 2010;4(3).

16. On S-L, Fouad AF, Park S-H. Treatment strategy for guided tissue regeneration in combined endodontic-periodontal lesions: case report and review. Journal of endodontics. 2009;35(10):1331-6.

17. Kim E, Song J-S, Jung I-Y, Lee S-J, Kim S. Prospective clinical study evaluating endodontic microsurgery outcomes for cases with lesions of endodontic origin compared with cases with lesions of combined periodontal-endodontic origin. Journal of Endodontics. 2008;34(5):546-51.

18. Jensen SS, Broggini N, Hiørting-Hansen E, Schenk R, Buser D. Bone healing and graft resorption of autograft, anorganic bovine bone and B-tricalcium phosphate. A histologic and histomorphometric study in the mandibles of minipigs. Clinical oral implants research. 2006;17(3):237-43.

\section{* Responsabilidad de Autor}

